\title{
Glandular Schwannoma: An Uncommon Variant of Schwannoma with Controversial Histogenesis
}

\author{
Andrea Saggini $^{\mathrm{a}} \quad$ Monia Di Prete ${ }^{\mathrm{a}}$ Federico D'Amico ${ }^{\mathrm{a}}$ Viviana Lora ${ }^{\mathrm{b}}$ \\ Augusto Orlandia

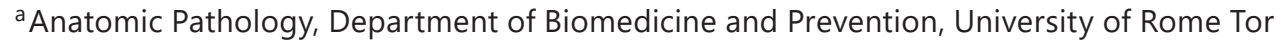 \\ Vergata, Rome, Italy; ${ }^{b}$ Division of Dermatology, San Gallicano Dermatological Institute, \\ Rome, Italy
}

\section{Keywords}

Glandular schwannoma - Pseudoglandular schwannoma - Peripheral nerve sheath tumor .

Neural crest

\section{Abstract}

Schwannomas are benign, encapsulated nerve sheath tumors arising from Schwann cells; several histologic variants of schwannoma have been described, including the exceedingly rare glandular schwannoma (GS). The histogenesis of the glands in GS (as well as in other peripheral nerve sheath tumors with a glandular component) remains unclear; no consensus exists as to whether glands should be interpreted as metaplastic elements or entrapped reactive glands. We report a case of GS with detailed immunohistochemical analysis of the epithelial component. We believe that our findings raise further questions regarding the histogenesis of glands in GS, casting doubts on the traditional distinction between true metaplasia and glandular entrapment. Further research is warranted in this regard.

\section{Introduction}

Schwannomas are benign, encapsulated nerve sheath tumors which are thought to arise from Schwann cells [1]; diagnosis of conventional schwannomas is fairly straightforward, resting on recognition of alternating hypercellular (Antoni A) and hypocellular (Antoni B) areas populated by bland $S 100(+)$ spindle cells $[1,2]$. 
Several histologic subtypes of schwannoma have been described, including ancient, cellular, epithelioid, plexiform, neuroblastoma-like, hybrid, and pseudoglandular variants [115]. A case of ancient schwannoma exhibiting features consistent with the exceedingly rare glandular variant of benign schwannoma (glandular schwannoma, GS) was recently described [1]. GS belongs to the heterogeneous group of peripheral nerve sheath tumors (PNSTs) with a glandular component, also referred to as glandular PNSTs $[3,8,16]$. The histogenesis of the glands in GS (and PNST) is unclear, as it remains controversial as to whether glands should be interpreted as truly metaplastic elements or as the result of entrapment of adjacent glands $[8,16]$. We report an additional case of GS with detailed immunohistochemical analysis of the epithelial component. We believe that our findings raise further questions regarding the histogenesis of glands in GS.

\section{Case Report}

A 57-year-old man with a history of multiple surgically excised lipomas presented with an asymptomatic, slowly growing subcutaneous nodule localized to the right neck. Upon physical examination, the subcutaneous tumor was mobile, being covered by normal skin, with a maximum diameter of $1.5 \mathrm{~cm}$.

Histologically, excisional biopsy of the nodule revealed a well-encapsulated neoformation consisting of haphazard fascicles of bland spindle cells with alternating hypercellular and hypocellular areas (Fig. 1a); occasional nuclear palisading could be observed in hypercellular areas (Fig. 1b), while hypocellular areas exhibited a loose fibromyxoid matrix with irregularly distributed thick-walled blood vessels. Mitotic figures or necrotic foci were not noted. Importantly, several gland-like structures could be observed throughout the neoplasm (Fig. 1a). Morphologic features of such glandular elements were variable; some of them appeared to have a lumen lined by a single layer of non-ciliated, polarized, cuboidal, or columnar epithelium with occasional pseudostratification (Fig. 1c), while others presented with narrower lumina surrounded by a stratified epithelium consisting of two to four layers of cuboidal cells with central nuclei (Fig. 1d). Neither cytological atypia nor mitotic activity were observed in any of the glandular structures.

On immunohistochemistry, the spindle cells were strongly and diffusely positive for S100 (Fig. 2a), while all the glandular structures were markedly positive for pancytokeratin (Fig. 2b-c). Subtyping of glands based on cytokeratin and p63 expression revealed that those consisting of a single layer of cuboidal/columnar epithelium were strongly positive for CK7 and CAM5.2 while lacking expression of p63 (Fig. 3a-c); in contrast, glands lined by stratified cuboidal nonpolarized epithelium had the opposite immunoprofile (Fig. 3a-c), with moderate expression of p63, tapering down from the periphery to the lumen. A thin outer myoepithelial cell layer could be highlighted only surrounding the CK7(+)/CAM5.2(+) glands by means of faint immunoreactivity for muscle-specific actin. Expression of CK20, TTF1, CDX2, or neuroendocrine markers chromogranin-A and synaptophysin was never observed. Staining for neurofilament highlighted rare bundles of axons scattered in the midst of the spindle cell population, while immunopositivity for epithelial membrane antigen (EMA) was noted throughout the peripheral capsule as well as at the level of the luminal border of each glandular element (Fig. 2d, 3d). A low proliferative activity was detected in both the neural and the epithelial cell population (Ki-67 labeling index of less than 10\%). Upon review of the morphologic and immunohistochemical findings, a diagnosis of benign GS was rendered. 

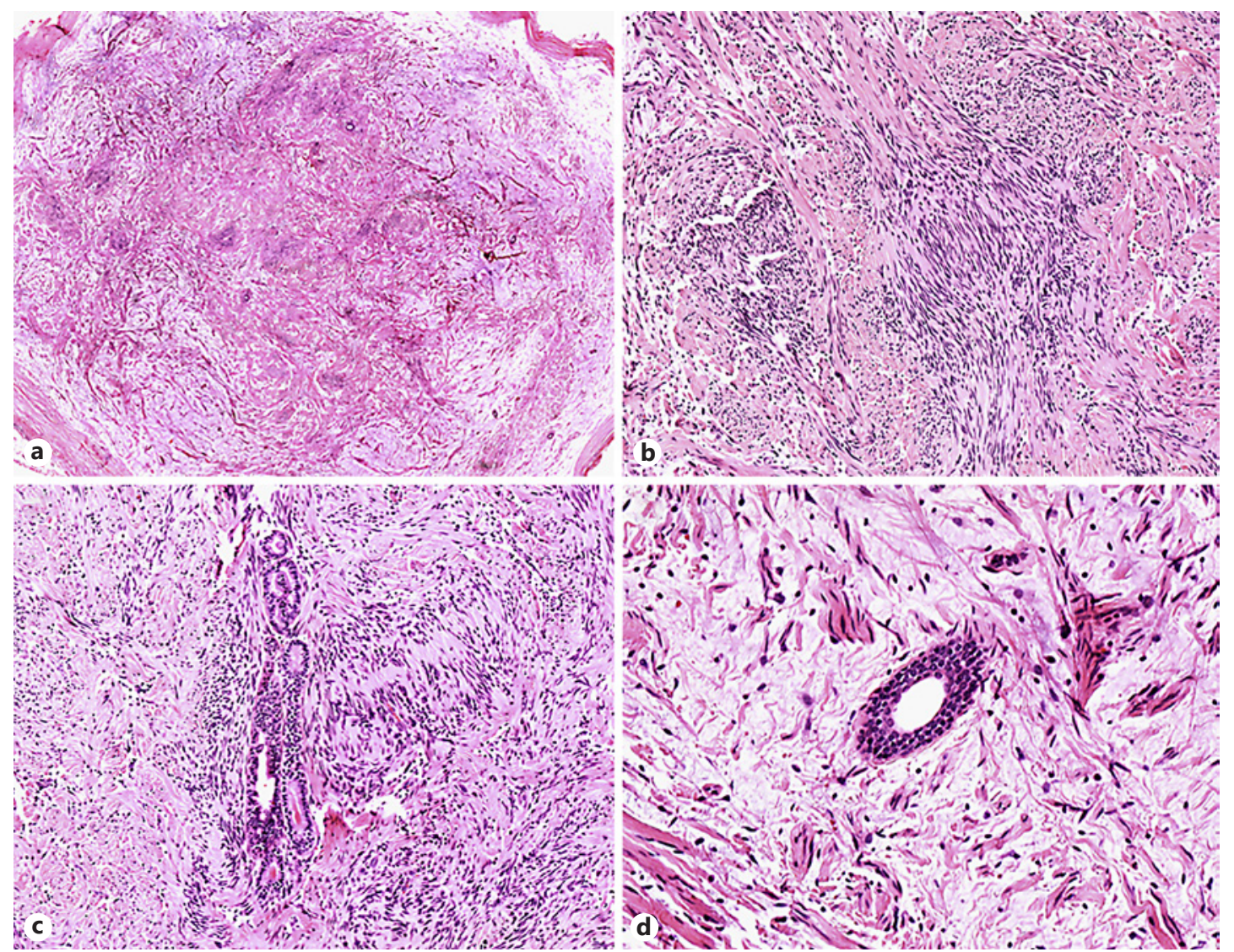

Fig. 1. a Encapsulated, well-circumscribed tumor composed of alternating hyper- and hypocellular areas populated by spindle cells; scattered glandular elements are seen. Hematoxylin and eosin. Original magnification, $\times 20$. b Haphazard fascicles of bland spindle cells with hints of palisading. Hematoxylin and eosin. Original magnification, $\times 100$. c Glands consisting of a single layer of polarized columnar epithelium with pseudostratification, surrounded by spindle cell proliferation. Hematoxylin and eosin. Original magnification, $\times 100$. d Immature glandular structures lined by three to four layers of cuboidal epithelial cells with central nuclei; no cytological atypia is noticed. Hematoxylin and eosin. Original magnification, $\times 200$.

\section{Discussion}

GS is a neuroepithelial neoplasm characterized by the presence of benign-looking epithelial glands randomly scattered within the spindle cell population of an otherwise conventional schwannoma [1, 2]. GS appears to be exceedingly rare; according to a recent review [1], prior to our case only 12 cases of GS had been reported in the available literature. It should be noted, however, that the actual frequency of GS may be underestimated, as at times the epithelial component may be scarcely and not homogeneously represented, thus requiring serial sections to become apparent. GS tend to have small size and superficial location. Similarly to conventional schwannomas, the association between GS and neurofibromatosis type I is weak to absent; the clinical course is indolent $[1,2,7,9]$.

Histologically, the epithelial component of GS exhibits an invariably benign morphology [1, 2]. Glandular lumina are usually lined by bland, non-ciliated cuboidal and/or pseudostratified columnar epithelium. Enteric goblet cell-like differentiation may be observed, with intra- and/ or extracellular mucin production; evidence of respiratory or intestinal differentiation has been 


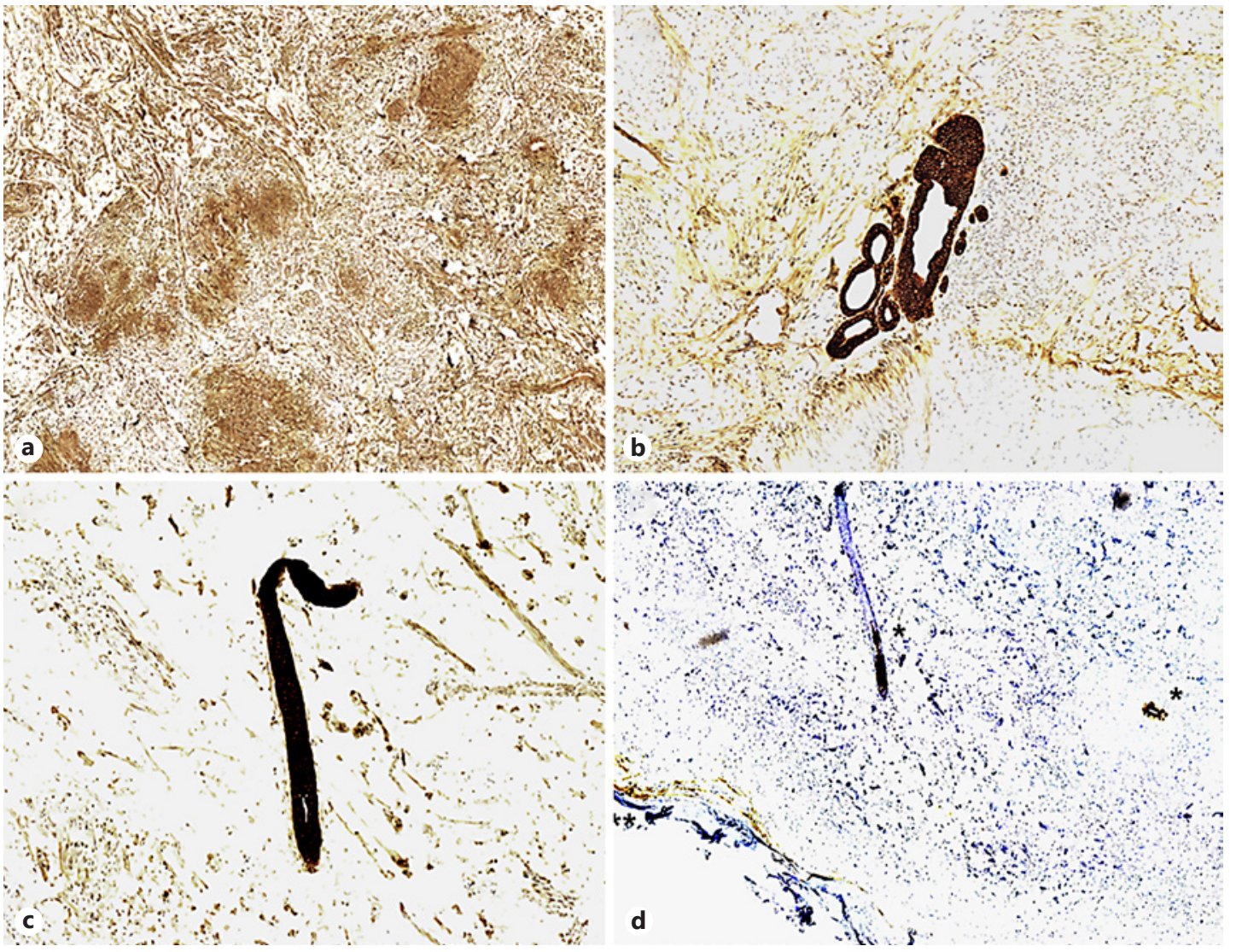

Fig. 2. a Strong and diffuse expression of $S 100$ by bland spindle cells. Original magnification, $\times 40$. b, c Glandular epithelium is strongly positive for pancytokeratin. Original magnification, $\times 100$. d Strong positivity of the glandular luminal border for EMA $\left(^{*}\right)$; milder expression of EMA is seen in the peripheral capsule $\left(^{* *}\right)$. Original magnification, $\times 40$.

occasionally described by means of electron microscopy $[5,6,8,9]$. On immunohistochemistry, the epithelial nature of glandular structures is confirmed by positivity for cytokeratins; as expected, the luminal border of glands stains positive for EMA, which also highlights the perineurial capsule encasing the schwannomatous proliferation. The neural spindled cell population stains with S100, as in conventional schwannoma [1,2]. Conflicting data have been reported concerning the presence of a muscle-specific actin-positive outer myoepithelial cell layer, as well as of single neuroendocrine cells scattered among cuboidal/columnar epithelium $[1,2,7,9,10]$.

GS is part of the broader group of glandular PNST, which also includes glandular neurofibroma and malignant PNST (MPNST) with glandular differentiation [16-20]. Glandular neurofibromas appear to be even less common than GS [17-19]; histologically, benign epithelial glands are observed lying within the neurofibromatous tissue of a conventional or plexiform neurofibroma. MPNST are known to hold a potential for heterologous differentiation, with glandular differentiation being one of the rarest forms $[16,20]$; glands may exhibit variable degrees of cytological and architectural atypia, ranging from benign glands to adenocarcinomatous elements $[8,16,20]$. The degree of glandular atypia does not appear to correlate with that of the spindle cell population; indeed, the presence of glandular differentiation is not thought to have an impact on MPNST behavior, with prognosis being solely related to the malignant spindle cell component $[16,20]$. 

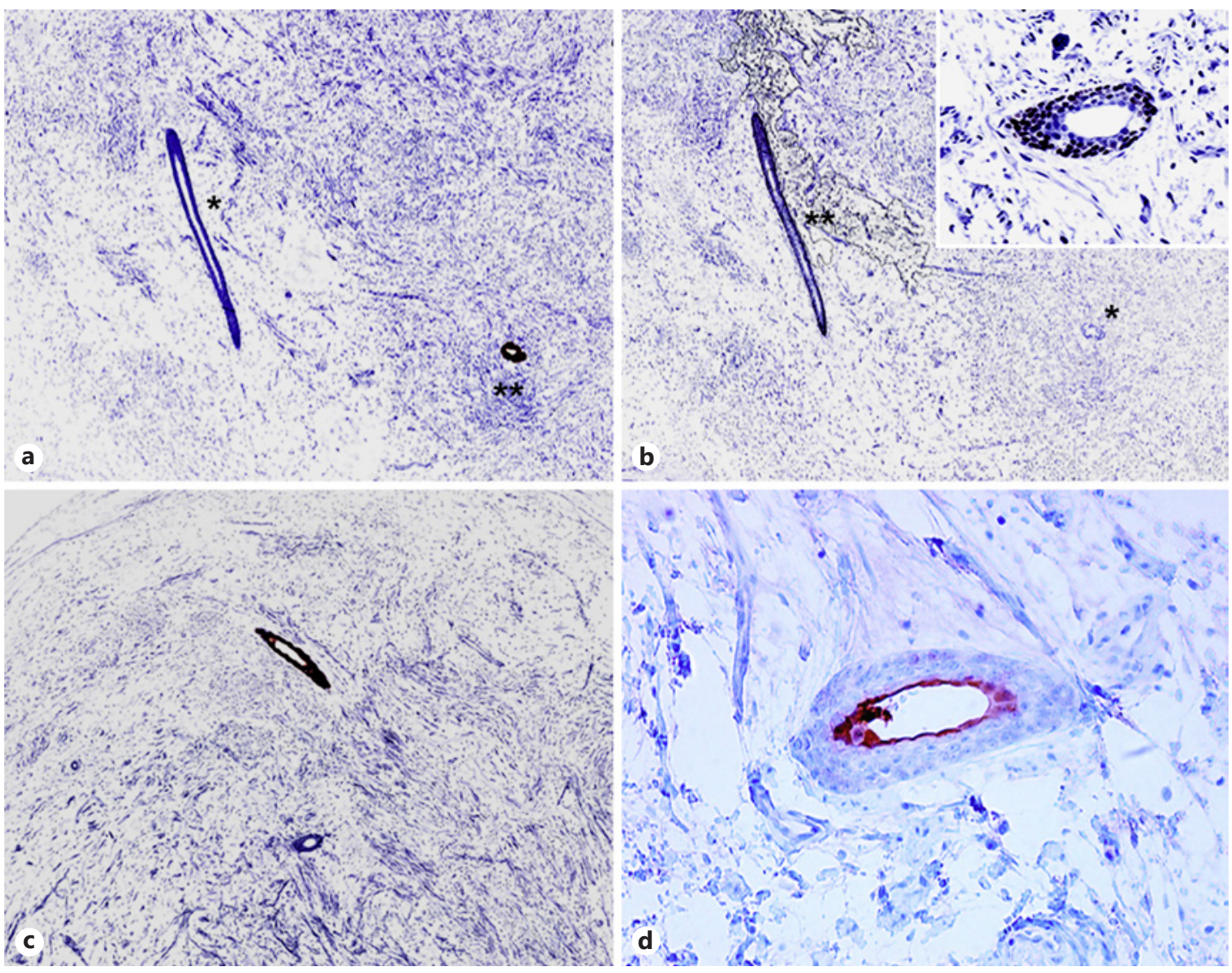

Fig. 3. a, b Differential pattern of expression of CAM5.2 (a) and p63 (b) distinguishes CAM5.2(+)/p63(-) simple glands $\left(^{*}\right)$ from CAM5.2(-)/p63(+) immature glands (**). Original magnification, x40. Inset Higherpower view of nuclear p63 expression by immature gland. Original magnification, $\times 200$. c Expression pattern of CK7 overlaps with that of CAM5.2. Original magnification, $\times 40$. $\mathbf{d}$ Positivity for EMA on the luminal border of an immature gland. Original magnification, $\times 200$.

The differential diagnosis of GS includes cytokeratin-positive malignant tumors (such as sarcomatoid carcinoma, carcinosarcoma, and synovial sarcoma, among the others) $[1,2,10$, 20], colonization of an otherwise benign schwannoma by metastatic adenocarcinoma [21], as well as pseudoglandular schwannoma (PGS) and mixed tumor of the soft tissues (MT) [11-15, 22]. PGS is an uncommon histologic variant of schwannoma characterized by gland-like structures as well as cystic spaces lined by epithelial-like cells of schwannian derivation [11-15]. Such epithelial-like cells appear to merge with surrounding spindled Schwann cells; immunohistochemistry confirms their schwannian nature as evidenced by positivity for S100 and lack of expression of cytokeratins and EMA. PGS was initially regarded as the "missing link" between conventional schwannoma and true GS, but pseudoglandular elements are now thought to be the consequence of intratumoral degenerative changes resulting in accumulation of proteinaceous fluid [11-13]. MT often exhibits cytokeratin-positive glandular structures within a variably S100-positive chondromyxoid stroma harboring hyalinized vessels, similarly to GS [22]. Nonetheless, strong and diffuse S100 expression as observed in the spindle cell component of GS is inconsistent with a diagnosis of MT.

The histogenesis of epithelial glands in GS remains unclear. It was initially suggested that these glandular structures might represent heterotopic ependymal glands, but such hypothesis 
was later refuted based on electron microscopic data [2, 4]. Likewise, no convincing evidence has ever been offered supporting an origin from primitive neural crest cells migrating along the peripheral nerves into the schwannian neoplasm $[10,20]$. At present, it is thought that glandular elements in GS may derive from true metaplasia of neoplastic Schwann cells, or rather be the result of entrapment of surrounding sweat or salivary glands (depending on the cutaneous or oral location of GS, respectively) by the enlarging tumor [2, 4, 7, 9, 10, 23]. Schwann cells may retain mesectodermal potential, thus being still capable of epithelial differentiation under exceptional conditions $[4,8,17,20]$. On the other hand, entrapped pre-existing glands may undergo reactive hyperplasia under the inductive stimuli from neoplastic Schwann cells. In this regard, several instances of reactive growth of epithelial cells in close apposition to neoplastic neural elements have been described, such as pseudoepitheliomatous reactive hyperplasia adjacent to neural granular cell tumor, plexiform schwannoma, traumatic and palisaded encapsulated neuromas, common and Spitz melanocytic nevi, and subgemmal neurogenous plaque [24].

Several authors have maintained that true metaplasia of neoplastic neural cells may be distinguished from entrapment of non-neoplastic glands based on a constellation of morphologic and immunohistochemical features $[2,4,7,9,10]$. Accordingly, evidence of respiratory or intestinal differentiation, immunohistochemical detection of neuroendocrine cells scattered within the glandular epithelium, as well as lack of muscle-specific actin-positive myoepithelial lining, would all be findings supporting a metaplastic origin from Schwann cells rather than reactive hyperplasia of pre-existing entrapped glands.

Our findings are at odds with such dichotomic vision; the present case was characterized by the coexistence of simple glands with a CK7 $(+) /$ CAM5.2(+)CK20(-)/p63(-) phenotype, surrounded by a thin layer of myoepithelial cells, and immature glandular elements consisting of two to four layers of CK7 (-)/CAM5.2(-)/CK20(-) cuboidal cells with central nuclei positive for $\mathrm{p} 63$. According to the above-mentioned criteria, the first gland population might represent entrapped glands, while the second gland population might be the result of immature metaplasia of schwannian cells. Importantly, the two types of glandular structures appeared to be haphazardly distributed and intermixed throughout the neoplasm, as if belonging to the same biologic process.

We believe that our findings suggest that the distinction between glandular metaplasia and entrapment in GS may be arbitrary. The question of whether epithelial cells in GS should be regarded as neoplastic elements, sharing at least part of the genetic abnormalities of neoplastic Schwann cells, or rather hyperplastic cells responding to the inductive influence of neoplastic Schwann cells, is still unresolved. Definitive evidence in this regard might result from comparative genetic assessment of the two cell populations in GS, following the example of MT, where the presence of the same oncogenic fusion gene has been proved in the nuclei of both mesenchymal and epithelial cell populations [22]. In the meantime, we agree with Holliday et al. [1] that all schwannomas with glands should be regarded as GS, regardless of circumstantial evidence pointing to true glandular metaplasia rather than gland entrapment.

In sum, we reported an additional case of the uncommon glandular variant of schwannoma. Differently from MPNST with glandular differentiation, GS is a benign neoplasm with an indolent course. Our findings question the feasibility of distinguishing between GS with true glandular metaplasia and entrapment of pre-existing glands by growing schwannoma. Further research is warranted to clarify the histogenesis of epithelial glands in GS.

\section{Statement of Ethics}

The authors have no ethical conflicts to disclose. Patient consent was not required for this publication. 


\section{Disclosure Statement}

The authors have no conflicts of interest to declare.

\section{Funding Sources}

The authors have no funding sources to declare.

\section{References}

1 Holliday AC, Mazloom SE, Coman GC, Kolodney MS, Chavan RN, Grider DJ. Benign Glandular Schwannoma With Ancient Change. Am J Dermatopathol. 2017 Apr;39(4):300-3.

2 Chuang ST, Wang HL. An unusual case of glandular schwannoma. Hum Pathol. 2007 Apr;38(4):673-7.

3 Woodruff JM. Peripheral nerve tumors showing glandular differentiation (glandular schwannomas). Cancer. 1976 May;37(5):2399-413.

4 Fletcher CD, Madziwa D, Heyderman E, McKee PH. Benign dermal Schwannoma with glandular elementstrue heterology or a local 'organizer' effect? Clin Exp Dermatol. 1986 Sep;11(5):475-85.

5 Brooks JJ, Draffen RM. Benign glandular schwannoma. Arch Pathol Lab Med. 1992 Feb;116(2):192-5.

6 Yoshida SO, Toot BV. Benign glandular schwannoma. Am J Clin Pathol. 1993 Aug;100(2):167-70.

7 Elston DM, Bergfeld WF, Biscotti CV, McMahon JT. Schwannoma with sweat duct differentiation. J Cutan Pathol. 1993 Jun;20(3):254-8.

8 Woodruff JM, Christensen WN. Glandular peripheral nerve sheath tumors. Cancer. 1993 Dec;72(12):3618-28.

9 Woodruff JM. Benign glandular schwannoma. Am J Clin Pathol. 1994 Apr;101(4):550-1.

10 Kim YC, Park HJ, Cinn YW, Vandersteen DP. Benign glandular schwannoma. Br J Dermatol. 2001 Nov; 145(5): 834-7.

11 Ferry JA, Dickersin GR. Pseudoglandular schwannoma. Am J Clin Pathol. 1988 Apr;89(4):546-52.

12 Deng A, Petrali J, Jaffe D, Sina B, Gaspari A. Benign cutaneous pseudoglandular schwannoma: a case report. Am J Dermatopathol. 2005 Oct;27(5):432-5.

13 Lisle A, Jokinen C, Argenyi Z. Cutaneous pseudoglandular schwannoma: a case report of an unusual histopathologic variant. Am J Dermatopathol. 2011 Jul;33(5):e63-5.

14 Sundarkrishnan L, Bradish JR, Oliai BR, Hosler GA. Cutaneous Cellular Pseudoglandular Schwannoma: An Unusual Histopathologic Variant. Am J Dermatopathol. 2016 Apr;38(4):315-8.

15 Ud Din N, Ahmad Z, Ahmed A. Schwannomas with pseudoglandular elements: clinicopathologic study of 61 cases. Ann Diagn Pathol. 2016 Feb;20:24-8.

16 Miki Y, Thway K. Malignant Peripheral Nerve Sheath Tumor with Divergent Glandular Differentiation. Int J Surg Pathol. 2017 Jun;25(4):310-3.

17 Oda Y, Hashimoto H, Tsuneyoshi M, Iwata Y. Benign glandular peripheral nerve sheath tumor. A case report. Pathol Res Pract. 1994 May;190(5):466-73.

18 Cuesta T, Estupiñán B, Salazar S, Carballosa E. [Neurofibroma with mucus-producing glands. Report of a case and literature review]. Rev Neurol. 1999 Feb;28(3):245-7. Spanish.

19 Joshi D, Gangane N, Kishore S, Vagha S. Unusual histological presentation in neurofibromas: two case reports. Cases J. 2008 Sep;1(1):188.

20 Galatian AA, Crowson AN, Fischer RJ, Yob EH, Shendrik I. Malignant peripheral nerve sheath tumor with glandular differentiation in a patient with neurofibromatosis type 1. Am J Dermatopathol. 2013 Dec;35(8):859-63.

21 Gazic B, Pizem J. Lobular breast carcinoma metastasis to a superficial plexiform schwannoma as the first evidence of an occult breast cancer. Am J Dermatopathol. 2011 Dec;33(8):845-9.

22 Antonescu CR, Zhang L, Shao SY, Mosquera JM, Weinreb I, Katabi N, et al. Frequent PLAG1 gene rearrangements in skin and soft tissue myoepithelioma with ductal differentiation. Genes Chromosomes Cancer. 2013 Jul;52(7):675-82.

23 Tarjan G. Schwannoma including epithelial elements mimicking pleomorphic adenoma of the submandibular gland on fine-needle cytology: the first case report. Diagn Cytopathol. 2015 May;43(5):395-8.

24 Ide F, Muramatsu T, Kikuchi K, Saito I, Kusama K. Oral plexiform schwannoma with unusual epithelial induction. J Cutan Pathol. 2015 Dec;42(12):978-82. 